$\begin{array}{cl}\begin{array}{cl}\text { Revue } \\ \text { de Ihistoire }\end{array} & \text { Revue de l'histoire des religions } \\ \text { des religions } & 4 \mid 2010 \\ & \text { Qu'est-ce qu'un « paysage religieux »? }\end{array}$

\title{
Y a-t-il un paysage religieux colonial ? Entre prescription, mimétisme et adaptation : les mécanismes de l'imitatio Romae
}

A Specifically Colonial Religious Landscape? Between Instruction, Imitation, and Adaptation: the Mechanisms of Imitatio Romae

\section{Audrey Bertrand}

\section{OpenEdition}

Journals

Édition électronique

URL : http://journals.openedition.org/rhr/7676

DOI : $10.4000 /$ rhr.7676

ISSN : 2105-2573

Éditeur

Armand Colin

Édition imprimée

Date de publication : 1 décembre 2010

Pagination : $591-608$

ISBN : 978-2200-92658-8

ISSN : 0035-1423

\section{Référence électronique}

Audrey Bertrand, «Y a-t-il un paysage religieux colonial ? Entre prescription, mimétisme et adaptation : les mécanismes de l'imitatio Romae », Revue de l'histoire des religions [En ligne], 4 | 2010, mis en ligne le 01 décembre 2013, consulté le 21 avril 2019. URL : http://journals.openedition.org/rhr/7676 ; DOI :

$10.4000 /$ rhr.7676 


\section{Y a-t-il un paysage religieux colonial? Entre prescription, mimétisme et adaptation : les mécanismes de l'imitatio Romae}

L'article propose une réflexion sur le paysage religieux des colonies, afin de mieux définir l'impact de ce statut juridique et politique sur celui-ci. L'examen de la charte de fondation de la colonie d'Vrso révèle que Rome constitue un modèle. Résultats de la nécessaire adaptation au contexte local, les différences observées soulignent aussi que la lex Vrsonensis (45 avant notre ère) reflète les évolutions les plus récentes du paysage religieux de Rome. Parallèlement, l'observation des phénomènes d'imitation au sein des colonies montre que le statut colonial peut aussi être valorisé par les élites locales dans la construction du paysage religieux de leur cité et, partant, éclaire la variété des processus qui rendent compte de la spécificité des sacra publica coloniaux.

\section{A Specifically Colonial Religious Landscape? Between Instruction, Imitation, and Adaptation : the Mechanisms of Imitatio Romae}

This article is an attempt to assess the impact of the legal and political status of the Roman coloniae on their religious landscape. The founding charter of the Vrso colonia is examined and reveals that Rome was a genuine model. The observed differences can be interpreted as necessary adaptations to the local context, and they emphasize how the Lex Vrsonensis (45 BC) reflected the latest evolutions of the Roman public religion. Moreover, studying imitation mechanisms within the coloniae shows that the religious landscape of a city could be constructed by the local elite in order to enhance the colonial status and provides precious insights into the various processes explaining the specific forms taken by the sacra publica in the Roman coloniae. 
Questionner la spécificité du paysage religieux des colonies s'appuie sur un double constat de départ. Le premier concerne le contexte au sein duquel doit être appréhendée la religion publique, qui ne se comprend que dans le cadre de la communauté civique qui la prend en charge, celle qui définit le calendrier des fêtes publiques, assure le bon déroulement des rites et désigne ceux qui les célébreront. Le second renvoie à la définition du statut colonial. Juridiquement assimilées à des quartiers de Rome, les colonies n'ont pas l'autonomie des municipes et, ce faisant, obéissent à un fonctionnement politique, juridique et administratif spécifique. La religion publique, partie intégrante des institutions de la cité, se conforme aux règles du droit romain. La combinaison de ces deux prémisses implique un nécessaire impact du fait colonial sur le paysage cultuel des cités, que les sources peinent cependant à faire ressortir face à la mosaïque hétérogène que composent les études monographiques.

C'est en effet souvent à travers une documentation éparse que se dévoile la « romanité » du fonctionnement de la religion publique dans les colonies. Quelques témoignages particulièrement suggestifs de la pertinence du modèle de Rome pour envisager la vie religieuse des colonies, en Italie ou dans l'Empire, sont ainsi sollicités par les historiens pour éclairer en quoi le fonctionnement de la religion publique de ces cités obéissait à des normes conformes à celles du droit romain. La représentation sur un relief de la colonie d'Alexandria Troas d'un flamine du divin Jules portant l'apex, coiffe distinctive des flamines de Rome, offre une image saisissante ${ }^{1}$. Les trois autels des colonies d'Ariminum, de Narbo et de Salona, dont les lois de dédicace se réfèrent explicitement à celles du temple de Diane sur l'Aventin, constituent eux aussi un corpus, certes réduit mais de premier ordre, pour appréhender la diffusion du modèle romain ${ }^{2}$. Signalons encore la réponse faite par les quindécemvirs de Rome aux décurions de Cumes qui avaient demandé l'approbation du collège quant à la désignation de leur nouveau prêtre de

1. Stefan Weinstock, Divus Iulius, Oxford, Clarendon Press, 1971, pl. 31.2.

2. Ariminum: CIL, XI, 361; Narbo: CIL, XII, 4333 (ILS, 112); Salona: CIL, III, 1933 (ILS, 4907). Voir Umberto Laffi, " La lex arae Iouis Salonitanae », Athenaeum 58 (1980), p. 119-127 (repris dans Umberto Laffi, Studi di storia romana e di diritto, Rome, s. n., 2001, p. 545-558). 
la Magna Mater ${ }^{3}$. À la fin du III $^{\mathrm{e}}$ siècle de notre ère, la colonie romaine continuait de se référer aux autorités romaines dans la gestion de ses prêtrises publiques.

En arrière-plan de cette documentation dispersée, les sources littéraires dessinent une toile de fond harmonieuse, où s'affirme tout autant le lien particulier qui unit les colonies à Rome que se dessine l'idée d'une régularité des paysages d'une colonie à l'autre. Ces textes sont bien connus et il n'est point besoin ici de s'y arrêter trop longuement. Dans le De Lingua Latina, Varron insiste sur l'identité entre Rome et les cités qu'elle a fondées, à travers la réplication du rite de fondation et, par conséquent, l'attribution aux colonies du titre d'urbes ${ }^{4}$. Deux siècles plus tard, Aulu-Gelle donne à ce rapport de similitude sa forme quasi définitive, aux yeux des Modernes, en définissant les colonies comme des « images réduites et en quelque sorte des reproductions » de « la grandeur et de la majesté du peuple Romain ${ }^{5} »$. Fonctionnant selon «les lois et les institutions du peuple romain et non pas celles de leur choix », les colonies accèdent grâce au compilateur du $\mathrm{II}^{\mathrm{e}}$ siècle au statut de «petites Rome» dans l'historiographie. Ces textes, parmi les plus explicites sur le paysage colonial - et pourtant loin de constituer l'ensemble du corpus sur ce sujet -, demeurent très vagues. La tension se révèle ainsi entre, d'un côté, des documents variés qui apparaissent comme les "balises-témoins » d'un fonctionnement juridique à explorer, mais dont la valeur heuristique demeure problématique, et, de l'autre, des textes dont le niveau de généralité reste trop élevé pour fonder le raisonnement.

Une telle étude ne peut en réalité faire l'économie d'un élargissement du champ problématique. À la mise au jour des contraintes juridiques qui régissent le fonctionnement de la religion publique dans les colonies doit s'ajouter la prise en compte d'autres mécanismes qui contribuent à construire la romanité du paysage religieux des colonies et à en faire l'un des lieux de l'imitatio Romae. Défini en termes de droit, le statut colonial peut aussi être investi par les agents locaux sur le terrain de l'identité et du prestige. Ainsi, une mise au point préalable concernant l'unique texte normatif dis-

3. CIL, X, 3698 (ILS, 4175).

4. Varron, De Lingua Latina, V, 143.

5. Aulu-Gelle, Noctes atticae, 16, 13, 8-9. 
ponible sur l'organisation de la religion publique dans une colonie de droit romain, la lex coloniae Genetiuae Vrsonensis, se révèle dans un premier temps indispensable. Une approche de terrain, à travers l'examen d'un document provenant de la colonie adriatique de Potentia, offrira dans un second temps un point de vue complémentaire sur l'impact du statut colonial, en tant qu'il est investi par les élites citadines.

\section{RetOUR SUR LA LEX COLONIAE GENETIUAE IULIAE : L'ESPRIT DES LOIS}

Le constat d'une organisation de la religion publique des colonies calquée sur celle de Rome, mais simplifiée, est largement partagé. Il s'appuie sur l'exégèse d'un texte normatif: la lex coloniae Genetiuae Vrsonensis. Cette loi de fondation, datée de 45 avant notre ère et « donnée » par Rome à la colonie césarienne d'Vrso en Bétique, comporte un certain nombre de dispositions relatives à l'organisation de la religion publique, toutes insérées au sein de paragraphes abordant des questions plus variées. Par exemple, les prescriptions détaillant l'organisation des jeux sacrés en l'honneur de la triade capitoline et de Vénus s'inscrivent dans un chapitre plus large consacré aux obligations financières des magistrats ${ }^{6}$. Cette lecture de la loi, qui met en relief la volonté de ses rédacteurs de dessiner un cadre d'action conforme au droit romain, se retrouve dans les nombreuses études qui lui ont été consacrées, tant pour le domaine religieux que pour celui du droit public ou pénal ${ }^{7}$. Récemment cependant, une révision de cette interprétation « romaniste » de la lex Genetiuae Iuliae a été entreprise, sous la plume de

6. Michael H. Crawford, Roman statutes, Londres, Institute of Classical Studies, University of London, 1996, vol. I, p. 393-454, chapitres 70 et 71 (nous indiquerons les chapitres de la loi en nous référant à cette édition).

7. Parmi les plus importantes: M. H. Crawford, Roman statutes, op. cit., p. 393-454; John Scheid, «Aspects religieux de la municipalisation. Quelques réflexions générales », dans Monique Dondin-Payre, Marie-Thérèse RaepsaetCharlier (éd.), Cités, municipes, colonies. Les processus de municipalisation en Gaule et en Germanie sous le Haut-Empire, Paris, Presses Universitaires de la Sorbonne, 1999, p. 381-423 ; la synthèse de Mary Beard, John North, Simon Price, Religions de Rome, trad. fr., Paris, Picard, 2006 (1998), p. 160 et 310; et l'étude d'Andrea Raggi, "Le norme sui sacra nelle leges municipales», dans Luigi Capogrossi Colognesi, Emilio Gabba (dir.), Gli statuti municipali, Pavie, IUSS Press, 2006, p. 701-721. 
Jörg Rüpke, au motif que si certaines dispositions renvoient sans conteste à une matrice romaine, elles ont largement été surévaluées aux dépens de prescriptions qui révèlent, elles, tout l'écart entre Rome et sa colonie. Dès lors, l'historien s'est attaché à établir deux faits : d'abord, que Rome n'a pas cherché à exporter le contenu de sa religion dans les colonies mais plutôt son « concept », et ensuite que les structures de la religion publique de la colonie d'Vrso, telles qu'elles sont fixées par la charte de fondation, ne présentent pas une version simplifiée de celles observables à Rome. Bien qu'il ne s'agisse ici ni de retranscrire en détail l'argumentaire fourni pour appuyer la démonstration, ni de trop le simplifier, il est cependant nécessaire d'en résumer les différentes étapes afin d'évaluer la pertinence sinon d'une lecture entièrement iconoclaste, du moins des inflexions très nettes qui sont proposées.

Le premier point ne nécessite guère que l'on s'y arrête longtemps car il y a en réalité un consensus clair sur le fait que Rome n'a jamais voulu imposer le «contenu » de sa religion dans les colonies ${ }^{8}$. Le second point ouvre en revanche un terrain d'examen plus vaste. Afin de remettre en cause la validité du modèle romain comme source de la loi de fondation, Jörg Rüpke propose un examen minutieux des prêtrises, envisageant successivement les fonctions qui leur incombent, l'organisation des collèges qui les regroupent et enfin les modalités de leur élection.

\section{Chômage technique chez les prêtres?}

La loi d'Vrso prévoit que la colonie devra compter deux collèges religieux, celui des pontifes et celui des augures. Selon Jörg Rüpke, leurs fonctions sont quasi inexistantes au regard des dispositions contenues dans la charte, à la différence de Rome où ces deux collèges religieux, les plus importants de la cité, bénéficient de prérogatives majeures ${ }^{9}$. L'absence dans le texte de la charte d'une liste

8. Jörg Rüpke, «Urban Religion and Imperial Expansion : Priesthoods in the Lex Ursonensis », dans Lukas de Blois, Peter Funke, Johannes Hahn (éd.), The Impact of Imperial Rome on Religions, Ritual and Religious Life in the Roman Empire, Leyde, Brill, 2006, p. 22. Les conclusions de John Scheid allaient déjà dans ce sens : J. Scheid, «Aspects religieux de la municipalisation», art. cité, p. 417-419.

9. J. Rüpke, «Urban Religion and Imperial Expansion », art. cité, p. 20. Sur les prérogatives du collège des pontifes, voir Françoise Van Haeperen, Le collège 
détaillant les différentes activités potentielles des pontifes est-elle en soi significative? Rien n'est moins sûr. La nature même des fonctions qu'ils assument à Rome ne se prête guère à un exposé circonstancié. Des deux champs principaux d'activité qui leur incombent, l'expertise en matière de droit sacré et la participation aux rites publics ${ }^{10}$, le premier déborde toute tentative de formulation englobante, qui plus est dans un texte de loi où la concision... fait loi. Les pontifes assument en effet un magistère sur l'ensemble des traditions cultuelles et sur le droit sacré, et c'est le plus souvent à la suite de demandes ponctuelles (d'origine publique ou privée) qu'ils interviennent dans la vie religieuse de la cité. Responsables également du maintien ou de la restauration de la pax deorum, ils pouvaient occasionnellement, quand un scrupule religieux surgissait, ordonner un certain nombre de rites. On le voit, rien là de suffisamment systématique ou précis pour être laconiquement consigné sur une tablette de bronze. La deuxième sphère d'action des pontifes, la participation aux rites publics, se prêterait a priori mieux à une inscription dans la charte de fondation. Si l'on a longtemps eu tendance à minimiser le rôle d' " acteurs cultuels » des pontifes, il apparaît que ceux-ci avaient en réalité une place de premier ordre dans nombre de fêtes régulières ${ }^{11}$. Aucun des chapitres de la lex Vrsonensis ne stipule en effet que les pontifes aient à prendre en charge une fête précise. Et pour cause : dans la mesure où le calendrier devait être fixé par les duovirs eux-mêmes dans les dix jours suivant leur entrée en charge, il était impossible aux rédacteurs de la charte de dresser une liste des activités cultuelles des pontifes ${ }^{12}$. En revanche, le chapitre 66 présente comme acquis la prise en charge de cérémonies publiques par les pontifes (« et cum ei pontific(es) augures sacra publica c(oloniae) G(enetiuae) I(uliae) facient »).

Quant aux augures, leur sphère d'activité ne pose guère de difficultés: "de auspiciis quaeque ad eas res pertinebunt augurum

pontifical ( $3^{e}$ s. a.C. $-4^{e}$ s. a.C.) : contribution à l'étude de la religion publique romaine, Bruxelles, Belgisch historish Instituut te Rome, 2002. Sur les augures, voir Jerzy Linderski, « The augural Law », Aufstieg und Niedergang der römischen Welt, II.16 3 (1986), Berlin-New York, Walter de Gruyter, 1986, p. 2146-2312.

10. Cette division, visible dans les sources littéraires, est acceptée et reprise par F. Van Haeperen, Le collège pontifical, op. cit., p. 215-423.

11. Notamment pour les fêtes liées au cycle naturel de l'année: ibid., p. 342-363.

12. Chapitre 64. 
iuris dictio iudicatio esto ${ }^{13} »$. La charte rappelle ainsi de manière rapide et efficace que les augures ont en charge les auspices. La formule « quaeque ad eas res pertinebunt », par son caractère allusif, suppose d'ailleurs un modèle implicite auquel se référer; lequel, sinon Rome ${ }^{14}$ ? Les arguments a silentio avancés par Jörg Rüpke semblent dès lors peu pertinents puisque le texte sous-entend luimême l'existence d'autres fonctions et prérogatives, bien que non mentionnées explicitement.

\section{Tradurre, tradire? L'organisation des collèges religieux}

L'organisation interne des deux collèges contribue elle aussi à mieux évaluer la validité du modèle romain. Les rédacteurs de la charte prévoient en effet que chacun comportera trois membres. Selon Jörg Rüpke, cette réduction drastique des effectifs en regard de la situation romaine révèle une fois encore tout l'écart qui sépare la colonie de Rome ${ }^{15}$. La miniaturisation des institutions, et plus largement la simplification des structures de la religion publique, contredit-elle l'idée d'une volonté des fondateurs coloniaux d'organiser la colonie sur un modèle romain?

La réduction des effectifs reposes sur une nécessaire mise en proportion de la taille des collèges à la taille de la cité concernée. À l'instar des sénats locaux qui, sauf exception, ne comptent jamais autant de membres que le Sénat romain ${ }^{16}$, les collèges religieux présentent logiquement des proportions réduites. Il semble d'ailleurs très significatif au contraire que la charte s'attache à préserver l'orga-

13. Chapitre 66.

14. On peut d'ailleurs remarquer, à la suite de Jerzy Linderski, l'emploi du terme « iudicatio » qui semblerait définir l'existence d'une « independant augural iudicatio in matters concerning the auspicia » : J. Linderski, "The augural Law », art. cité, p. 2160-2161. Même si l'auteur démontre la fausseté de cette impression, il est intéressant de retrouver chez Cicéron, et donc pour Rome même, à la fois l'emploi de termes similaires et une impression semblable lorsque l'avocat rapporte l'action des augures après l'erreur commise par Ti. Gracchus : Cicéron, De divinatione., 2, 74.

15. J. Rüpke, «Urban Religion and Imperial Expansion », art. cité, p. 20.

16. Les sénats locaux comptaient pour la plupart 100 membres : Richard Duncan-Jones, The Economy of Roman empire : Quantitative Studies, Cambridge, Cambridge University Press, 1974, p. 283-287. Selon François Jacques, le minimum légal devait se situer entre 50 et 100 : François Jacques, «CIL, XI, 2650 et la population de Saturnia », Zeitschrift für Papyrologie und Epigraphik 99 (1993), p. 218 , n. 6 . 
nisation collégiale de ces prêtrises. Comme l'affirme Jörg Rüpke, trois membres est bien un minimum pour former un collège mais derrière ce chiffre, on lit moins un quelconque caractère symbolique, voire factice, de ces collèges, que précisément la volonté de maintenir la collégialité telle qu'elle est en vigueur dans les institutions de la capitale ${ }^{17}$. Il est très probable que le chiffre de trois soit entendu comme un minimum plutôt que comme un effectif fixe et définitif. Les collèges de prêtres, comme les curies, étaient appelés à se développer en miroir de l'accroissement démographique potentiel de la cité. Quelques données chiffrées semblent confirmer cette hypothèse : à une date certes bien postérieure ( $\mathrm{IV}^{\mathrm{e}}$ siècle de notre ère), la colonie de Thamugadi compte quatre pontifes et quatre augures ${ }^{18}$.

\section{La lex Vrsonensis, miroir déformant de Rome ou miroir grossissant?}

L'insistance sur l'indigence des fonctions réelles des pontifes, et dans une moindre mesure sur l'amputation des prérogatives des augures, ainsi que l'importance accordée au caractère restreint des structures de la religion publique dans la colonie amènent Jörg Rüpke à une conclusion plus globale sur la place respective des prêtres et des magistrats dans la colonie : se donnerait à voir dans la charte de fondation d'Vrso une cité où la hiérarchie traditionnelle entre le ius des sacra et des sacerdotes d'une part, et le ius des magistrats d'autre part, telle qu'exprimée par Cicéron ${ }^{19}$, serait abolie; une cité, donc, où l'antériorité et la supériorité des sacra et du prêtre ont disparu. Les deux signes les plus visibles en seraient tout à la fois l'ampleur des fonctions religieuses des magistrats et le mode d'élection des pontifes et des augures, en tous points similaire à celui des autres détenteurs de charges publiques. Là résiderait in fine l'écart entre Rome et sa colonie.

Qu'en est-il? D'abord, nous l'avons vu, la supposée inactivité partielle des pontifes et des augures ne résiste pas à un examen détaillé

17. Des inscriptions provenant de Cirta et de Cuicul confirment l'organisation collégiale des pontifes et des augures : existence d'un magister pontificum dans la première (CIL, VIII, 7123), d'un mag(ister) augur [u]m dans la seconde (AE, 1912, 154).

18. CIL, VIII, 2403 (ILS, 6122).

19. Cicéron, De lege agraria, 2, 69. 
de la charte. Pareillement, l'adaptation des sacerdoces et des collèges à la taille d'une colonie de vétérans ne peut être utilisée pour démontrer leur caractère non romain. Cependant, l'on ne peut contredire certaines des conclusions apportées par la relecture de Jörg Rüpke quant à l'organisation de la religion publique de la colonie : les prérogatives religieuses des magistrats sont vastes et il n'est pas contestable que le mode d'élection des prêtres diffère très sensiblement de celui en vigueur à Rome. Et en ce sens, l'on peut légitimement conclure à la fin de la supériorité du ius des sacra et des sacerdotes. Cependant, ces constatations ne contribuent pas à notre sens à invalider la pertinence du modèle romain dans l'élaboration de la charte; on peut y lire bien au contraire le reflet des évolutions que connait Rome au cours de la première moitié du $\mathrm{I}^{\mathrm{er}}$ siècle avant notre ère, soit pendant les décennies qui virent l'élaboration progressive et discontinue des différentes rubriques qui composent la lex Vrsonensis.

La place éminente des magistrats dans la sphère religieuse publique, et parallèlement celle des activités religieuses au sein de leurs prérogatives, apparaissent clairement à la lecture de la charte $^{20}$. Mais cette répartition des tâches offre un visage entièrement romain. Comme l'a montré récemment John Scheid, les magistrats occupent à Rome le premier plan dans le fonctionnement de la religion publique ${ }^{21}$. En ce sens, l'organisation de celle-ci dans la colonie d'Vrso se conforme fidèlement aux principes du droit romain et à la répartition de la gestion des sacra publica entre prêtres et magistrats. D'une certaine manière, en raison du caractère simplifié qui définit les institutions coloniales, ces dernières se présentent comme un miroir grossissant de Rome : ce qui se dessine en filigrane dans la capitale, où la densité des prêtrises empêche peutêtre parfois d'appréhender les activités religieuses quotidiennes et multiples des magistrats, se manifeste plus immédiatement dans la charte d'Vrso. Derrière une façade épurée, l'architecture se dévoile plus clairement.

Plus troublantes apparaissent les dispositions relatives au mode d'élection. Abordées dans les chapitres 67 et 68 de la loi, les moda-

20. Chapitres 64, 69, 70, 71, 128.

21. John Scheid, "Les activités religieuses des magistrats romains », dans Rudolph Haensch, Johannes Heinrichs (éd.), Herrschen und Verwalten. Der Alltag der römischen Administration in der Hohen Kaiserzeit, Cologne, Böhlau, 2007, p. 126-144. 
lités d'élection des prêtres sont claires : elles sont semblables à celles en vigueur pour les magistrats. On doit donc supposer que les pontifes, les augures et les autres prêtres éventuels étaient élus par l'assemblée du populus. Il y a là un écart certain avec la situation romaine : jusqu'à la fin du $\mathrm{II}^{\mathrm{e}}$ siècle avant notre ère, le recrutement des prêtres s'y effectuait en effet par simple cooptation. Au tournant du $\mathrm{II}^{\mathrm{e}}$ et du $\mathrm{I}^{\mathrm{er}}$ siècle avant notre ère, en vertu de la lex Domitia (104-103 avant notre ère), un système complexe se met en place qui introduit une part de contrôle populaire dans la désignation des détenteurs des charges religieuses. Plus exactement, la désignation des membres des quatre collèges majeurs (pontifes, augures, décemvirs et septemvirs) se conforme désormais au mode d'élection en vigueur pour le seul pontifex maximus depuis la moitié du $\mathrm{III}^{\mathrm{e}}$ siècle avant notre ère : une assemblée spéciale composée de dix-sept tribus seulement était convoquée et votait ${ }^{22}$. À la lumière de ces évolutions du paysage religieux romain, les modalités d'élection des pontifes et des augures à Vrso se présentent comme l'adaptation, voire l'aboutissement, d'un processus en cours à Rome. Il est significatif que les rédacteurs de la charte se soient attachés à conserver l'esprit des institutions romaines, dont l'évolution était nette depuis un demi-siècle. Sous la République en effet, prêtres et magistrats supérieurs agissent de manière solidaire mais séparée et les institutions s'attachent à marquer cette distinction nette entre les deux provinces au sein desquelles ils interviennent ${ }^{23}$. À partir du II ${ }^{\mathrm{e}}$ siècle avant notre ère cependant, une évolution historique nette se dessine, bien qu'heurtée, qui aboutit sous Auguste à la « fin de la séparation radicale du pouvoir religieux et du pouvoir civil ${ }^{24} \gg$. Parmi les autres étapes marquantes de cette évolution, outre la lex Domitia, on peut signaler la lex Labiena de 63 qui rétablit le mode d'élection des prêtres des quatre collèges majeurs par les tribus après son abrogation par Sylla, mais aussi l'augmentation parallèle par César

22. John Scheid, «Le prêtre et le magistrat. Réflexions sur les sacerdoces et le droit public à la fin de la République », dans Claude Nicolet (dir.), Des ordres à Rome, Paris, Publications de la Sorbonne, 1984, p. 243-280. Nous simplifions ici une évolution plus heurtée puisqu'il y eut d'abord un retour à l'ancien système sous Sylla, puis une réactivation du système mis en place par Cn. Domitius Ahenobarbus par l'action du tribun de la plèbe T. Labienus (63 avant notre ère).

23. J. Scheid, «Le prêtre et le magistrat », art. cité, p. 243-280 (particulièrement p. 272).

24. Ibid., p. 279. 
du nombre de prêtres et de magistrats, et, sous Auguste, la réorganisation des sacerdoces entre sénateurs et chevaliers qui soumet de fait les prêtres à des critères de recrutement censitaires, c'est-à-dire identiques à ceux des magistrats. C'est donc tout à la fois l'aboutissement de ce processus et son sens profond qui se font jour dans la loi d'Vrso.

Dans cette perspective enfin, l'on ne peut que souligner les échos que la réforme du calendrier romain de Jules César trouve dans la loi de fondation coloniale. Les duovirs prennent en charge chaque année l'établissement du calendrier. Ici encore, le décalage avec Rome semble manifeste puisque le calendrier y était établi par les pontifes. Plusieurs éléments amènent à nuancer ce constat. À travers la proclamation mensuelle par le rex sacrorum, le jour des nones, des fêtes fixes à venir, ceux-ci manifestaient certes leur pouvoir sur la gestion du temps ${ }^{25}$. Cela dit, depuis la publication du calendrier en 304 avant notre ère, si cette annonce mensuelle du rex sacrorum perdurait, elle représentait désormais une survivance archaïque et en partie symbolique. En outre, un grand nombre de fêtes mobiles étaient fixées à Rome par des magistrats et non par les pontifes. L'attribution par la lex Vrsonensis de la maîtrise du temps aux décurions ne constitue donc pas une innovation. L'aspect le plus intéressant de cette disposition se révèle néanmoins au regard du contexte immédiat de la déduction de la colonie césarienne d'Vrso. Elle intervient en 45 avant notre ère, soit un an après la réforme du calendrier par Jules César. Si celle-ci, voulue par le dictateur, ne privait pas le collège pontifical de la prérogative d'établir le calendrier mensuel ni de procéder à l'intercalation, elle limitait cependant grandement son autonomie en la matière. En alignant le calendrier romain sur l'année solaire, le dictateur instaurait un système dont la régularité empêchait par avance les manipulations pontificales, puisque désormais un seul jour devait être intercalé tous les quatre ans et avant les cinq derniers jours de février ${ }^{26}$. Si les pontifes réussirent malgré tout à contrevenir aux prescriptions césariennes, en intercalant tous les trois ans au lieu de tous les quatre ans - au bénéfice d'une ambiguïté lexicale de l'édit -, la réforme du calendrier entendait bien les déposséder en

25. F. Van Haeperen, Le collège pontifical, op. cit., p. 216-237.

26. J. Rüpke, Kalender und Öffentlichkeit, op. cit., p. 380-383. 
grande partie de leurs prérogatives en cette matière. Il est d'ailleurs significatif que les sources s'accordent toutes ou presque sur le fait que César a agi dans sa réforme du calendrier en tant que dictateur et non en tant que grand pontife ${ }^{27}$. Ainsi, en octroyant aux premiers magistrats de la colonie la charge de fixer le calendrier annuel, la loi de fondation de la colonie reflète ici aussi, et entérine, une tendance lourde et profonde de la redistribution des tâches à Rome entre sphère civile et sphère religieuse ${ }^{28}$.

L'adaptation et la simplification nécessaires des structures de la religion publique des colonies engendrent des différences réelles entre le modèle romain et sa transposition. Elles ne peuvent certes être minimisées. Pourtant, bien plus qu'elles ne remettent en question la romanité du cadre religieux colonial, elles traduisent les évolutions les plus récentes du fonctionnement de la religion publique de Rome, au moment même où la charte est rédigée. En ce sens le paysage religieux colonial se fait le miroir grossissant mais non déformant de celui de Rome.

Néanmoins, le contenu de la charte révèle aussi en creux l'étendue des informations qui sont tues et, partant, la difficulté de fonder une étude de la religion publique des colonies uniquement sur des textes juridiques, par nature concis et formulaires. Les études de cas se présentent comme un complément nécessaire à la compréhension du paysage religieux. Si celles-ci offrent la possibilité de confronter des dispositions normatives aux modalités de leur application, elles permettent surtout d'envisager la variété des effets du statut colonial ; celui-ci ne se résume pas à une série de mesures contraignantes qui encadrent la vie religieuse des cités, il constitue aussi

27. Jörg Rüpke conclut lui aussi que la réforme du calendrier est à attribuer à César dictateur et non à César grand pontife (ibid.). Voir également Zvi Yavetz, César et son image. Des limites du charisme en politique, trad. fr., Paris, Les Belles Lettres, 1990, p. 126-128. L'argument principal allant dans ce sens est le fait que la réforme s'appuie sur un édit du dictateur. Rien n'empêche d'imaginer, comme le souligne Françoise Van Haeperen, que César ait pu consulter le collège pontifical mais cela fut fait «pour la forme » : F. Van Haeperen, Le Collège pontifical, op. cit., p. 223. Position inverse dans Roger Seguin, "Remarques sur les origines des pontifes romains : Pontifex Maximus et Rex sacrorum », dans Danielle Porte, Jean-Pierre Néraudeau (éd.), Hommages à Henri le Bonniec : Res Sacrae, Bruxelles, Latomus, 1988 [coll. Latomus, 201], p. 405-418.

28. Auguste attendit d'être grand pontife pour procéder à sa réforme calendaire (peut-on y lire une confirmation paradoxale de l'attitude contraire adoptée par César?). Voir John Scheid, « Auguste et le grand pontificat. Politique et droit sacré au début du Principat », Revue d'histoire du droit 77 (1999), p. 1-19. 
un ensemble de ressources symboliques à leur disposition. Autrement dit, l'impact du statut colonial sur la vie religieuse d'une cité ne doit pas s'évaluer uniquement à l'aune de l'application stricte de la règle juridique mais s'envisager parallèlement comme une identité potentiellement investie et valorisée par les agents locaux.

\section{L'autel du Clipeus Virtutis de Potentia}

\section{La restitution de l'autel}

La colonie de Potentia, fondée en 184 avant notre ère sur la côte adriatique de l'Italie centrale ${ }^{29}$, a livré en ce sens un document particulièrement riche. Un autel dédié par les sévirs augustaux de la colonie présente sur sa face principale une réplique du bouclier des vertus offert par le Sénat et le Peuple romain à Auguste le 16 janvier 27 avant notre ère et exposé dans la Curie $^{30}$. Celuici est connu depuis la moitié du XIX ${ }^{\mathrm{e}}$ siècle grâce à un fragment trouvé dans les environs de Porto Recanati, mais la découverte d'un second fragment muré sous le porche du Palazzo Leopardi au début des années 2000 a permis d'en compléter la restitution ${ }^{31}$. Sous la dédicace, incomplète, est représenté le clipeus uirtutis soutenu par deux Victoires ailées. Une procession, au sein de laquelle peut être identifié un licteur, occupe la moitié inférieure du décor. L'autel, sans appartenir à la catégorie des autels monumentaux, présente des dimensions relativement importantes : 1,50 m de large et 1,20 m de haut au minimum ${ }^{32}$.

Le caractère lacunaire de la dédicace, dont seuls les noms incomplets de deux sévirs augustaux sont connus, empêche de déterminer avec certitude à qui était consacré l'autel et la date de son érection dans la colonie. L'on sait que la diffusion du bouclier des vertus

29. Tite-Live, 39, 33, 10; Vell., 1, 15.

30. $R G D A, 34,2$.

31. Le premier fragment, aujourd'hui perdu, a été publié dans le CIL (CIL, IX, 5811, ILS, 82), le second fragment par Simona Antolini, « L'altare con il clipeus uirtutis da Potentia », Picus 24 (2004), p. 9-28 et «Potentia », Supplementa Italica 23 (2007), p. 174-176, $\mathrm{n}^{\circ} 2$.

32. René Ginouvès, Anne-Marie Guimier-Sorbets, «Les autels de l'antiquité classique : problèmes de description », dans Robert Étienne, Marie-Thérèse Le Dinahet (éd.), L'espace sacrificiel dans les civilisations méditerranéennes de l'Antiquité, Paris, de Boccard, 1991, p. 277-290. 
connaît deux grandes phases, d'abord immédiatement après l'événement de 29 avant notre ère, puis au lendemain de la mort d'Auguste qui avait vu la mise en place d'une réplique du bouclier d'or sur le mausolée de l'empereur défunt ${ }^{33}$. Simona Antolini a donc proposé de dater l'autel entre le dernier quart du $\mathrm{I}^{\mathrm{er}}$ siècle avant notre ère et le premier quart du $\mathrm{I}^{\text {er }}$ siècle de notre ère ${ }^{34}$. Plusieurs éléments conduisent à identifier un autel destiné au culte des Lares publics ou des Lares Augusti et du Genius Augusti. Les similitudes des décors des autels du uicus Sandaliarius et du Belvédère à Rome, tous deux autels compitalices, sont un premier argument dans ce sens ${ }^{35}$. En outre, depuis longtemps déjà, il a été suggéré que les sévirs augustaux, prêtres semi-publics, étaient chargés hors de Rome du culte des Lares et du Génie d'Auguste tel qu'il avait été mis en place dans la capitale par l'empereur à partir de 12 avant notre ère.

\section{Du tempo national au tempo local : la colonie en représentation}

La présence à Potentia d'un autel décoré du bouclier des vertus constitue un élément de premier ordre dans l'étude des mécanismes de l'imitatio Romae dans le paysage religieux des colonies. Il s'agit là d'inscrire architecturalement l'un des signes majeurs du pouvoir augustéen. La datation de l'autel, son décor et la réactivité des élites municipales traduisent la volonté de faire vivre la colonie au rythme du calendrier romain : c'est tout autant à une temporalité désormais universelle qu'à un lieu matrice que se rattache la cité.

La comparaison de la situation de la colonie adriatique avec celle d'Arles offre plusieurs clefs de lecture du statut colonial. La colonie de Narbonnaise avait en effet reçu dès 26 avant notre ère, et des mains mêmes de l'empereur, une réplique monumentale, en marbre, du bouclier des vertus de la Curie. À Potentia, rien ne laisse supposer une intervention du pouvoir central dans la mise en place de l'autel au bouclier. Sa réalisation traduit ainsi la maî-

33. Henner von Hesberg, Silvio Panciera, Das Mausoleum des Augustus : der Bau und seine Inschriften, Munich, C. H. Beck, 1994, p. 118.

34. S. Antolini, «Potentia », art. cité, p. 174-176, n 2.

35. Sur l'autel du Belvédère: Paul Zanker, «Der Larenaltar im Belvedere des Vatikans ", Mitteilungen des Deutschen Archäologischen Instituts, Römische Abteilung 76 (1969), p. 205-218. Dédicace de l'autel du uicus Sandaliarius : CIL, VI, 448 (ILS, 3614). 
trise des modèles urbains de la part des commanditaires. Elle se lit notamment dans la présence sur l'autel de deux Victoires ailées soutenant le bouclier, qui rappellent inévitablement la mise en scène adoptée dans la Curie romaine : on sait en effet que le clipeus est venu y rejoindre l'autel et la statue de la Victoire qui avaient été consacrés peu de temps après le triple triomphe des 13, 14 et 15 août $29^{36}$. L'intégrité du message que véhicule le bouclier des vertus est comprise et préservée. Celui-ci n'est en rien réduit au rôle de support d'inscription et garde, au contraire, toute sa signification originelle. Parce qu'il porte une citation tangible et lumineuse de l'espace architectural et politique de la capitale, et parce qu'il est conçu en dehors de toute intervention extérieure à la colonie, l'autel de Potentia constitue un exemple clair du rôle des initiatives locales dans l'élaboration d'un paysage urbain où Rome se donne à voir comme modèle et référence.

Ce cas d'étude se révèle d'autant plus intéressant qu'il éclaire le rapport actif que les élites locales entretiennent aux modèles diffusés par le pouvoir central et, partant, la complexité des mécanismes de l'imitation où deux échelles s'imbriquent étroitement, locale et nationale. L'examen du clipeus de Potentia montre en effet de manière évidente les variations introduites localement par rapport à l'archétype romain. En premier lieu, la forme de l'objet elle-même diffère du bouclier d'or installé dans la Curie : bouclier de marbre sculpté sur la face antérieure d'un autel, il ne s'agit pas d'un élément de décor autonome dans le paysage urbain de la colonie. Plus significativement, le texte inscrit à l'intérieur du bouclier présente des modifications importantes dans la titulature augustéenne et dans les abréviations choisies. Celui du Sénat romain a été restitué comme suit :

Senatus | Populusque Romanus | Imp(eratori) Caesari diui f(ili) Augusto | co(n)suli VII dedit | clupeum uirtutis clementiae | iustitiae pietatis erga $\mid$ deos patriamque.

Celui de Potentia se présente ainsi :

S(enatus) P(opulus)q(ue) R(omanus) | Augusto dedit | clupeum uirtutis | [c]le[me]nti[ae, ius]t[itiae], | [pietatis erga deos] | patr[iam]q(ue).

36. Dion Cassius, 51, 22, 1. 
Les variations entre les inscriptions des boucliers des vertus (mausolée d'Auguste, autel du Belvédère, Arles, Potentia) ont depuis longtemps été soulignées et expliquées. Le cas d'Arles notamment, où le texte mentionne le $\mathrm{VIII}^{\mathrm{e}}$ consulat et non le $\mathrm{VII}^{\mathrm{e}}$, révèle l'actualisation de la titulature de l'empereur afin de la faire coïncider avec la date (26 avant notre ère) à laquelle la colonie reçut le bouclier des mains d'Auguste. Sur l'autel du Belvédère, la mention du grand pontificat révèle là aussi la mise à jour de la titulature de l'empereur, alors que l'absence de chiffre pour les autres éléments (acclamations impériales, consulats, puissances tribuniciennes) trahit l'embarras des commanditaires devant ces éléments évolutifs du cursus augustéen ${ }^{37}$. Le texte du bouclier de Potentia pourrait s'inscrire dans une problématique similaire : on remarque l'absence de la totalité des éléments de la titulature de l'empereur, uniquement désigné comme Auguste ${ }^{38}$. Pourtant, cette explication ne rend qu'imparfaitement compte du texte inscrit dans le bouclier de Potentia. On constate en effet que le lapicide a délibérément omis d'insérer la mention de la filiation divine, pourtant présente dans les trois autres répliques du bouclier - et, surtout, élément intangible de la titulature augustéenne -, ne nécessitant donc aucune mise à jour. Tout suggère en réalité que les commanditaires ont avant tout privilégié la concision : les modifications partielles du texte du bouclier répondent essentiellement à des contraintes d'espace puisque le champ épigraphique ne mesure que 21 centimètres et que la lisibilité exigeait donc que la titulature soit réduite à sa plus simple expression. Autrement dit, ce n'est pas l'autel qui a été conçu autour du décor du clipeus uirtutis mais bien le motif iconographique du clipeus uirtutis qui a été adapté à la conception d'ensemble de la face principale du monument. On mesure ici toute la différence entre le bouclier d'Arles, réplique presque parfaite de celui installé à l'entrée du Mausolée d'Auguste, conçu à Rome et offert à la colonie par le Prince, et l'autel de Potentia d'initiative locale. La procession des sévirs augustaux y occupe au moins la moitié de la surface, et le bouclier se trouve ainsi encadré par la représentation des commanditaires; au-dessus par l'inscription de leurs noms, en dessous par la figuration de leur procession. Les membres de ce col-

37. Augusto Fraschetti, Rome et le Prince, Paris, Belin, 1994, p. 310.

38. Ibid. 
lège traduisent donc par l'érection de cet autel leur capacité à adopter le langage du pouvoir de manière dynamique et non passive. Surtout, la mise en scène de la face antérieure de l'autel révèle que celui-ci participe d'une stratégie d'auto-représentation et de promotion personnelle à l'échelle de la cité. L'imitation ne se conçoit pas sans l'adaptation aux exigences du contexte local, urbanistiques et architecturales notamment.

Par les écarts qui séparent le «bouclier» de Potentia de celui de la Curia Iulia et par la comparaison que l'on peut mener avec celui de la colonie d'Arles se dessine un processus d'imitation complexe qui mêle chronologie nationale et chronologie locale d'un côté, conformité recherchée et enjeux personnels de l'autre. Les sévirs augustaux sont à l'origine du choix du bouclier comme motif iconographique principal de l'autel, et l'étude du texte de l'inscription se révèle la plus significative pour comprendre les mécanismes à l'œuvre : l'absence de toute titulature pour désigner Auguste sur le bouclier de Potentia traduit la prééminence des contraintes locales et la capacité des commanditaires à utiliser le langage du pouvoir sans le dénaturer. Dans ce processus mimétique, Rome conserve l'initiative puisque le pouvoir a pris soin de diffuser par différents biais l'image du clipeus uirtutis et ainsi a suscité la fabrication de répliques. Mais la chronologie locale, peut-être la création du collège des sévirs augustaux, a fourni l'occasion de faire écho à l'événement romain. On mesure ici de quelle façon une colonie peut elle-même prendre en charge la mise en scène dans le paysage religieux du lien particulier qu'elle entretient à l'Vrbs en dehors de toute contrainte d'ordre juridique.

Être colonie se définit avant tout par un ensemble de règles qui organisent sans doute le fonctionnement de la communauté civique selon les normes du droit public romain. À ce titre, et plus que d'autres cités encore, les colonies réaffirment que le cadre de la ciuitas est l'unité de base pour la compréhension des religions publiques. Le statut colonial ne se résume cependant pas à l'obligation de suivre un ensemble de contraintes institutionnelles, ni le lien qui unit ces cités à Rome à l'application de prescriptions venues d'en haut. Les mécanismes qui rendent efficient le statut colonial sont plus complexes et plus riches, les modes de domination plus euphémisés et intériorisés qu'ostensiblement directifs. Mesurer l'impact du statut juridique sur le paysage religieux ne doit donc 
pas se limiter à un recensement des preuves de l'application ou de la non-application d'une règle : l'effectivité d'une contrainte légale ne se juge pas seulement au fait qu'on lui obéisse passivement, elle s'évalue tout autant à l'aune des actions qui sont orientées en fonction d'elle ${ }^{39}$. Dans cette perspective, la notion de paysage religieux, en associant la matérialité des formes de la religion romaine à leur dimension élaborée et perçue, se fait l'écho d'une identité coloniale qui relève tout autant du droit formel que de représentations suscitées de concert par le pouvoir central et par les élites locales. À l'instar du droit, le paysage religieux se situe entre contrainte et ressource pour les habitants qui incorporent à son contact et forgent à son moyen une identité coloniale tout à la fois construite et prescrite.

audreybertrand@aol.com

39. Pierre Lascoumes, Évelyne Séverin, «Le droit comme activité sociale : pour une approche wébérienne des activités juridiques », Droits et société 9 (1988), p. 171-193. 